\title{
Revista Colombiana de

\section{Capítulo 10. Utilidad del mapeo tridimensional en la ablación de la taquicardia ventricular fascicular (corazón sano)}

\author{
Chapter 10. Usefulness of three-dimensional mapping \\ in fascicular ventricular tachycardia ablation \\ (healthy heart)
}

\section{Guillermo Mora}

Universidad Nacional de Colombia, Bogotá, Colombia

Fundación Santafé de Bogotá, Bogotá, Colombia

Hospital Universitario Clínica San Rafael, Bogotá, Colombia

Recibido el 17 de noviembre de 2015; aceptado el 18 de enero de 2016

\section{Introducción}

La taquicardia ventricular monomórfica se asocia comúnmente con cardiopatía estructural, especialmente isquémica o dilatada. Sin embargo, en un $10 \%$ de pacientes puede suceder en corazones sanos ${ }^{1}$ y se conoce como taquicardia ventricular idiopática. La forma más frecuente se da en el tracto de salida del ventrículo derecho ${ }^{2}$. Por su parte, las taquicardias ventriculares idiopáticas del ventrículo izquierdo pueden clasificarse de acuerdo con su mecanismo, como sensibles a verapamilo (reentrada), a adenosina (actividad gatillada) o a propranolol (automatismo) ${ }^{1}$; sin embargo también pueden clasificarse por su origen anatómico (fasciculares, tracto de salida, origen en senos de Valsalva, continuidad mitro-aórtica, etc.).

La taquicardia ventricular fascicular es la forma más frecuente de taquicardia ventricular idiopática del ventrículo izquierdo y se caracteriza por inducción con estimulación auricular y morfología de bloqueo de rama derecha con eje superior $^{3}$, además de su sensibilidad al verapamilo, como otro rasgo importante ${ }^{4}$. Esta taquicardia se origina en la zona del fascículo póstero-inferior, si bien se han descrito, aunque con menor frecuencia (menos del $10 \%$ de los casos), algunas que se generan en el fascículo antero-superior y en el septum superior $^{5,6}$.

La taquicardia ventricular fascicular se manifiesta comúnmente en adultos jóvenes, predominantemente varones ${ }^{7}$. $\mathrm{El}$ mecanismo es una macrorreentrada entre el fascículo posterior y el tejido de Purkinje anormal o miocardio adyacente con conducción decremental ${ }^{8}$. Durante la taquicardia, la conducción anterógrada ocurre en el tejido anormal Purkinje/ miocardio y la conducción retrógrada sobre el fascículo posterior $^{8}$. No obstante, algunos autores sugieren que el fascículo posterior no es necesario en el circuito dado que se puede realizar ablación exitosa sin producir bloqueo fascicular posterior ${ }^{9,10}$. En el estudio del sustrato anatómico se ha 
encontrado que en algunos pacientes existen falsos tendones o bandas fibro-musculares, aunque su papel exacto no es conocido. Se considera que la conducción en estos tejidos o el estiramiento que producen en la red de Purkinje, pueden llevar a las condiciones necesarias para la iniciación de la taquicardia ${ }^{11}$.

El tratamiento médico de esta patología se ha fundamentado en verapamilo, beta-bloqueadores y bloqueadores de canales de potasio con resultados contradictorios ${ }^{12}$, de ahí que muchos autores consideren que el tratamiento de elección sea la ablación por radiofrecuencia. Nakagawa fue el primero en demostrar que la ablación guiada por el potencial de Purkinje más precoz, era exitosa y no había relación con el tiempo más precoz de activación ventricular. El éxito del procedimiento es cercano al $90 \%^{7,8}$.

\section{Utilidad del mapeo tridimensional}

La reconstrucción electroanatómica y el mapeo tridimensional permiten una localización precisa del sistema de conducción del ventrículo izquierdo en su fascículo posterior y la localización de las lesiones de radiofrecuencia en relación con esta estructura (fig. 1). Cabe resaltar que en esta patología la ablación no se dirige al sitio más precoz de activación ventricular sino a la presencia del potencial de Purkinje más precoz en taquicardia ${ }^{7}$, en unos casos, o en otros por la presencia de un potencial pre-Purkinje ${ }^{11}$.

Otro grupo de pacientes en quienes el mapeo tridimensional puede ser útil es el grupo en el cual no es posible inducir la taquicardia durante el estudio electrofisiológico ${ }^{13}$. Una publicación reciente demostró que una lesión linear perpendicular al eje largo del ventrículo izquierdo en la mitad inferior del septum, entre la mitad y los dos tercios de distancia entre la base y el ápex, producía ablación exitosa. Las lesiones se extendían por 1,2 a 2,2 cm y se guiaban por la presencia

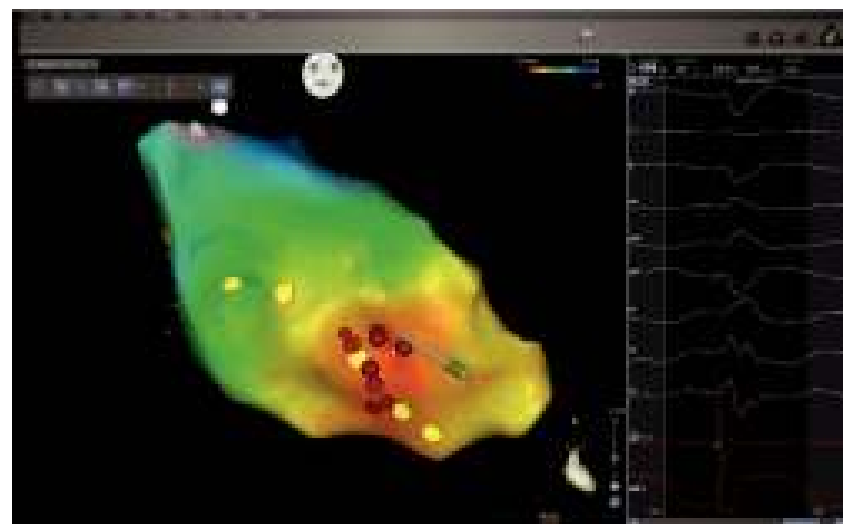

Figura 1 Reconstrucción electroanatómica del ventrículo izquierdo (CARTO) con localización del fascículo posterior (círculos amarillos), con lesiones de radiofrecuencia (círculos rojos) que cruzan el fascículo en forma perpendicular. Como en otras patologías arrítmicas, existen pacientes en quienes no se desea la exposición a los rayos X. Un estudio reciente con 19 pacientes que presentaban taquicardia ventricular idiopática utilizó ecocardiograma intracardiaco y mapeo tridimensional sin fluoroscopia. Los autores lograron $100 \%$ de éxito en la ablación; $11 \%$ de los pacientes tenían taquicardia ventricular fascicular ${ }^{13}$. de potenciales de Purkinje. En el seguimiento de 16 meses no se reportaron recurrencias en los pacientes intervenidos ${ }^{14}$.

El uso del mapeo tridimensional ha servido para obtener una mejor definición de la zona de conducción lenta. En un estudio con 10 pacientes se localizó la zona de conducción lenta, caracterizada por la presencia de un potencial diastólico, y la zona del sistema de conducción, definida por el potencial de Purkinje. La ablación fue dirigida al área de intersección de estas dos zonas y se logró terminar la taquicardia ventricular de manera exitosa ${ }^{15}$.

\section{Recomendaciones}

\section{Clase I}

- Se recomienda el mapeo tridimensional para guiar ablación por radiofrecuencia en pacientes con taquicardia ventricular fascicular en quienes se desea disminuir la exposición a los rayos $\mathrm{X}$ (nivel de evidencia $\mathrm{C}$ ).

- Se recomienda el mapeo tridimensional para guiar ablación por radiofrecuencia en pacientes no inducibles con taquicardia ventricular fascicular (nivel de evidencia C).

- Se recomienda el mapeo tridimensional para guiar ablación por radiofrecuencia en pacientes con recaída luego de ablación convencional (nivel de evidencia C).

\section{Clase II A}

- Puede recomendarse el mapeo tridimensional para guiar ablación por radiofrecuencia como estrategia inicial en pacientes con taquicardia ventricular fascicular.

\section{Bibliografía}

1. Lerman BB, Stein KM, Markowitz SM. Mechanism of idiopathic ventricular tachycardia. J Cardiovasc Electrophysiol. 1997; 8:571-83.

2. Rodríguez LM, Smeets JL, Timmermans C, Wellens HJ. Predictors for successful ablation of rigth and left-sided idiopathic ventricular tachycardia. Am J Cardiol. 1997;79:309-14.

3. Zipes DP, Foster PR, Troup PJ, Perdersen DH. Atrial induction of ventricular tachycardia: reentry versus triggered automaticity. Am J Cardiol. 1979;44:1-8.

4. Belhassen $B$, Rotmensh $\mathrm{HH}$, Laniado $\mathrm{S}$. Response of recurrent sustained ventricular tachycardia to verapamil. $\mathrm{Br}$ Heart J. 1981;46:679-82.

5. Ohe T, Shimomura K, Aihara N, et al. Idiopathic sustained left ventricular tachycardia clinical and electrophysiological characteristics. Circulation. 1988;77:560-8.

6. Shimoike E, Ueda N, Maruyama T, Kaji Y. Radiofrequency catheter ablation of upper septal idiopathic left ventricular tachycardia exhibiting left bundle branch block morphology. J Cardiovasc Electrophysiol. 2000;11:203-7.

7. Nakagawa H, Becleman KJ, Mc Clelland JH, et al. Radiofrequency catheter ablation of idiopathic left ventricular tachycardia guided by a Purkinje potential. Circulation. 1993;88: 2607-17.

8. Nogami A, Naito S, Tada H, et al. Demostration of diastolic and presystolic Purkinje potentials as critical potentials in a macroreentry circuit of verapamil-sensitive idiopathic left ventricular tachycardia. J Am Coll Cardiol. 2000;36:811-23.

9. Morishima I, Nogami A, Tsuboi H, Sone T. Negative participation of the left posterior fascicle in the reentry circuit of verapami- 
le-sensitive idiopathic left ventricular tachycardia. J Cardiovasc Electrophysiol. 2012;23:556-9.

10. Maeda S, Yokoyama Y, Nogami T, et al. First case of left posterior fascicle in a bystander circuit of idiopathic left ventricular tachycardia. Can J Cardiol. 2014;30:1460.e11-3.

11. Thakur RK, Klein GJ, Sivaram CA, et al. Anatomic substrate for idiopathic left ventricular tachycardia. Circulation. 1996; 93:497-501.

12. Ohe T, Aihara N, Kamakura S, et al. Long-term outcome of verapamil sensitive sustained left ventricular tachycardia in patients without structural heart disease. J Am Coll Cardiol. 1995;25:54-8.
13. Lamberti F, Di Clemente F, Remoli R, Bellini C, De Santis A, Mercurio $\mathrm{M}$, et al. Catheter ablation of idiopathic ventricular tachycardia without the use of fluoroscopy. Int J Cardiol. 2015; 190:338-43.

14. Lin D, Hsia HH, Gerstenfeld EP, et al. Idiopathic fascicular left ventricular tachycardia: linear ablation lesion strategy for noninducible or nonsustained tachycardia. Heart Rhythm. 2005; 2:934-9.

15. Chu J, Sun Y, Zhao Y, et al. Identification of the slow conduction zone in a macroreentry circuit of verapamil-sensitive idiopathic left ventricular tachycardia using electroanatomic mapping. J Cardiovasc Electrophysiol. 2012;23:840-5. 